\title{
Design and Realization of Intelligent Phase-controlled System for Permanent Magnetic Actuator of AC Contactor
}

\author{
Chen Ya bin *, Zhang Zhen tao, Li Qiang, Zou Ping Guo \\ SuZhou Nuclear Power Research Institute Co., Ltd., China
}

\begin{abstract}
When the AC contactor switches the capacitor bank, the phase angle of the power grid is random, so the electromagnetic transient process will generate large inrush current and overvoltage, causing large disturbance to the power system. A set of AC contactor with permanent magnetic actuator (PMA) and its intelligent phase selection control system are designed to solve this problem. Firstly, the PMA is designed. The dynamic characteristics of the actuator are simulated and analyzed in ANSOFT to meet the performance requirements of phase selection control. Then the principle of phase selection control and the strategy of switching capacitor bank are analyzed. Based on this, the software and hardware of intelligent phase selection control system are designed, including phase selection control module, IGBT driver module and coil discharge module. Finally, the experimental verification is carried out on the designed AC contactor. The test results show that the phase selection errors of the designed intelligence system are within $1^{\circ}$, and the contactor closing and opening time deviations are within $0.5 \mathrm{~ms}$, which means the system can effectively realize the closing and opening control at the specified phase angle.
\end{abstract}

\section{Introduction}

With the rapid development of China's economy and society and the continuous improvement of people's living standards, the demand and dependence of electricity is also increasing. By the end of 2018, the total installed capacity of full-scale power generations and the national total electricity consumption have reached 1.90 billion kilowatts and 6.84 trillion kilowatt hours respectively, a year-on-year increase of $6.5 \%$ and $8.5 \%$. Since more than $60 \%$ of the energy consumed by the power grid is inductive, a large number of parallel capacitors are required as reactive power compensations and power factor improvements. However, the magnitude of the load in the power grid is a variable that changes dynamically with time, therefore, the reactive power that needs to be compensated is not a fixed value. This means that a considerable number of switch devices are required to be ready to switching power capacitor banks of different capacities. As a kind of switch device suitable for frequent switching, AC contactors are widely used in switching power capacitor banks and their performance are directly related to power quality and grid stability.

Due to the random phase angle of the grid voltage, the electromagnetic transient process during the closing operation will cause a large disturbance to the power system when the $\mathrm{AC}$ contactor is switching the capacitor bank, and the maximum 80 100 times over-voltage and inrush current can be generated, causing damage to the switch itself, shortening the life of the switch, and causing malfunction of the relay protection [1]. Therefore, it is necessary to develop an intelligent $\mathrm{AC}$ contactor with phase-controlled technology. Phase-controlled technology refers to an intelligent control method that can control the contactor to close or open at the optimal phase angle of the grid voltage and current signal according to the nature of the load, which can effectively weaken the switching transient electromagnetic effect and improve the breaking reliability and voltage stability to prevent the switch from re-igniting due to arcing during opening.

Literature [2]-[8] analyzed and studied the intelligent control and phase selection of AC contactors, and obtained certain research results. In this paper, a PMA of the monostable $\mathrm{AC}$ contactor and its intelligent phasecontrolled system are designed. Some key parameters of the dynamic mechanism were simulated and verified in Ansoft, and then the hardware and software of the phasecontrolled system were designed respectively. Finally, the experimental verification was carried out to realize the optimal phase control of the contactor when switching the capacitor bank, which allows the contactor to operate accurately at a specified phase angle.

\section{Design and analysis of permanent magnetic actuator}

The PMA of the AC contactor is a complex multi-field coupling system, including electric, magnetic, mechanical and other fields. When the coil of the operating mechanism is energized, the current in the coil gradually rises, and the magnetic field generated by the current is superimposed by the permanent magnet, so the electromagnetic force is gradually increased. The

\footnotetext{
* Corresponding author: chenyabin2@cgnpc.com.cn
} 
contactor moves upward with the moving iron core when the electromagnetic force overcomes the gravity of the moving iron core and the springs' resistance. At this time, the spring begins to be compressed, and the reaction force rises. If the electromagnetic force is too small or the spring reaction coefficient is too large, the speed of the moving contact may decrease even to negative thus leads to the failure of PMA closing. Therefore, factors such as spring coefficient, coil current, closing speed and time should be comprehensively considered and analyzed in the simulation to meet the parameters of the vacuum tube. The common AC contactor permanent magnet operating mechanism is a double E-type magnetic system, as shown in Figure 1. This structure is a monostable permanent magnet operating mechanism, which mainly includes a coil, a static iron core, a permanent magnet, a moving iron core and the reaction spring set on the static iron core.

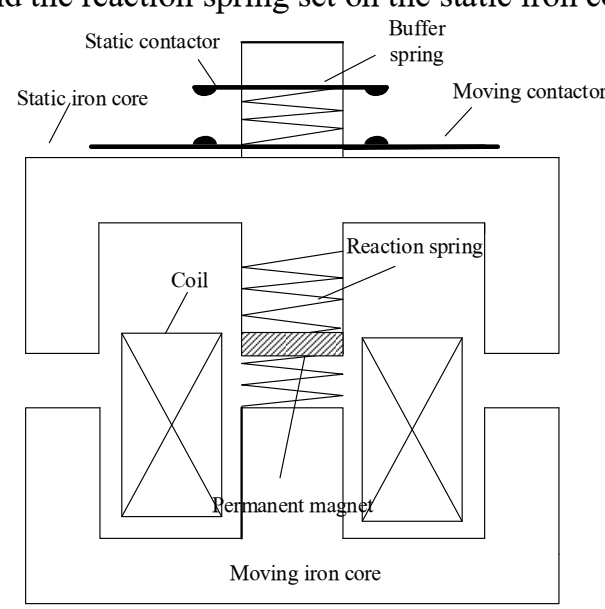

Fig.1 Double E-type permanent magnet AC contactor operating mechanism

\subsection{Finite Element Modeling}

The model of the PMA designed in Ansoft is shown in Figure 2. It is mainly composed of four parts: moving iron core, static iron core, permanent magnet, and coil and Band region. Band is used to indicate the range of the moving iron core. After the simulation model is built, the next step is to define and assign the material, add the excitation source, set the boundary conditions, add the spring reaction force and set the mesh. The coil is excited by the circuit that comes from Maxwell Circuit Editor, as shown in Figure 3.

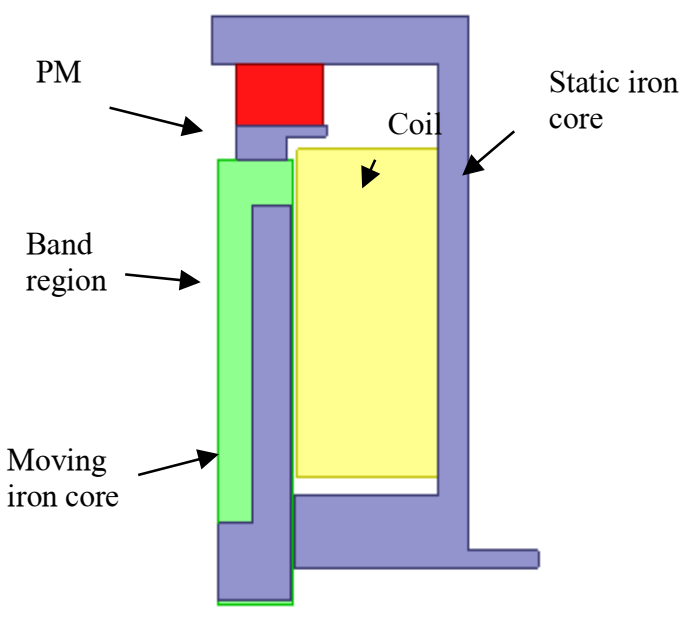

Fig.2 Dimensional simulation model of contactor PMA

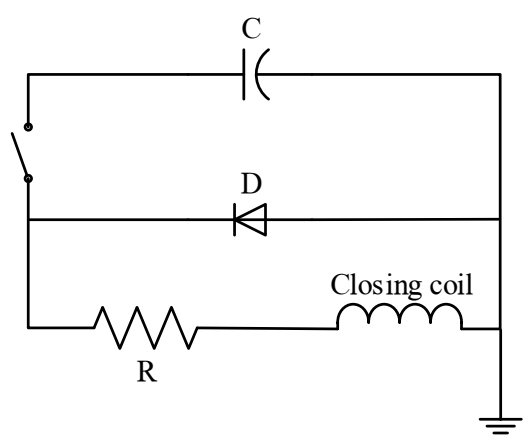

Fig.3 Ansoft excitation source applied circuit

\subsection{Simulation analysis}

Table 1 shows the relevant parameters of the designed PMA. After constructing the model in Ansoft and inputting the following parameters, the static simulation analysis without external excitation is first carried out. In the results toolbox of Ansoft, the holding force of the PMA at the closing position is calculated of $1051 \mathrm{~N}$. The suction force of the permanent magnet to the moving core is about $93.8 \mathrm{~N}$ at the opening position, which is smaller than the spring reaction force and can be kept in the opening position.

Table.1.parameters for PMA

\begin{tabular}{|c|c|}
\hline Coil turns & $\mathbf{2 3 2 9}$ \\
\hline Coil resistance $(\Omega)$ & 27.2 \\
\hline Thickness of PM $(\mathrm{mm})$ & 11 \\
\hline Capacitor $(\mathrm{F})$ & 0.001 \\
\hline Reaction spring coefficient & 105.72 \\
\hline Opening spring coefficient & 32.91 \\
\hline
\end{tabular}

The next step is to carry out the Then the dynamic simulation is carried out and analyst. Observe the dynamic parameter curve of the PMA through the Results toolbox in Ansoft, the current of the coil and the displacement and velocity of the moving iron core are presented in Figures 4 and 5, wherein the green line represents the current in the coil, the red line represents the motion velocity curve, 
and the blue line represents the moving iron core displacement curve. It can be seen that the closing time of the magnetic mechanism is $9 \mathrm{~ms}$, and the closing action time is $13 \mathrm{~ms}$, since the moving distance of the moving iron core is $8 \mathrm{~mm}$, so the average closing speed is about $0.62 \mathrm{~m} / \mathrm{s}$, and the peak current is about $5.5 \mathrm{~A}$ when closing; The interval time between electrifying and moving of the iron is about $5 \mathrm{~ms}$, and the opening action time is about $5.7 \mathrm{~ms}$, so the average opening speed is $1.4 \mathrm{~m} / \mathrm{s}$, and the peak current in the coil when opening is about $0.85 \mathrm{~A}$.

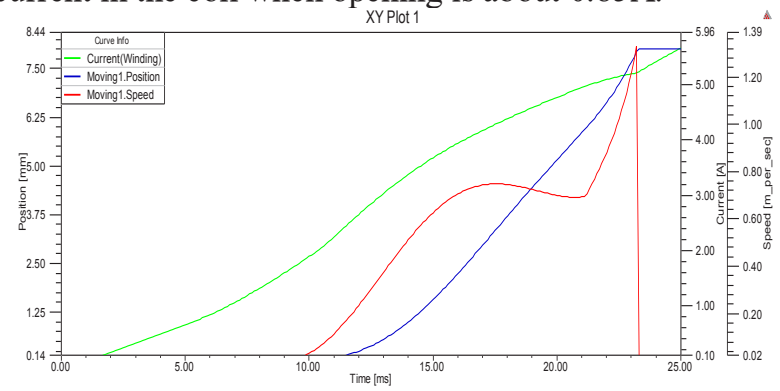

Fig.4 The curve of displacement velocity and current when closing

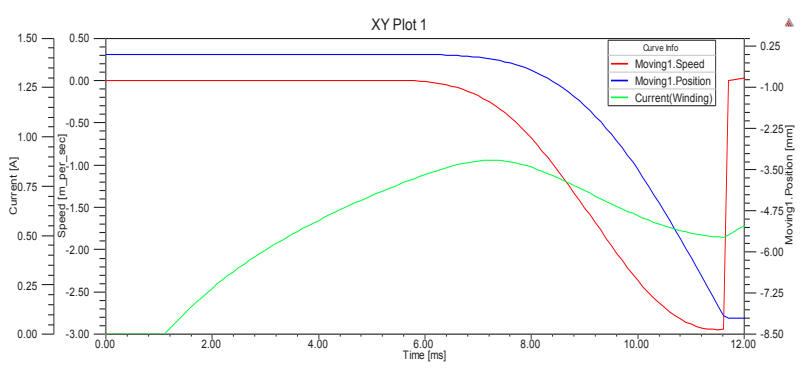

Fig.5 The curve of displacement velocity and current when opening

\section{Phase selection control principle and strategy}

The key to phase selection control technology is to accurately extract the zero crossing point of the grid signal, and use this as the reference zero point and calculate the delay time required to close or open at the specified phase angle according to a certain algorithm. The extracting of zero crossing point mainly includes hardware method and software method. This paper will concentrate on former method.

The specific implementation is as follows: 1)send the reference voltage or current signal to the high-precision zero-crossing comparator through the signal processing circuit; 2)extract the zero-crossing point by comparing with the zero potential, and then send the point to the external interrupt pin of the microprocessor; 3)turn on the external interrupt immediately when the closing or opening command is received; 4) set the zero-crossing point of the reference signal as the reference zero point of the phase-selection closing or opening, and calculate the delay time that required for the contactor to operate at the optimal opening or closing phase angle; 5) send a signal to the drive circuit and discharge the coil at the same time; 6)complete the phase-selection operation of the contactor. The full workflow is shown in Figure 6.
The magnitude of the inrush current and overvoltage generated by the AC contactor when switching the power capacitor is related to the initial phase angle of the power supply, and increases with the initial angle in the range of $0 \sim 90^{\circ}$. The inrush current and overvoltage are the smallest when the closing phase angle is $0^{\circ}$ or $180^{\circ}$ [9], so the contactor is designed to close at the zero crossing point to suppress the inrush current and overvoltage.

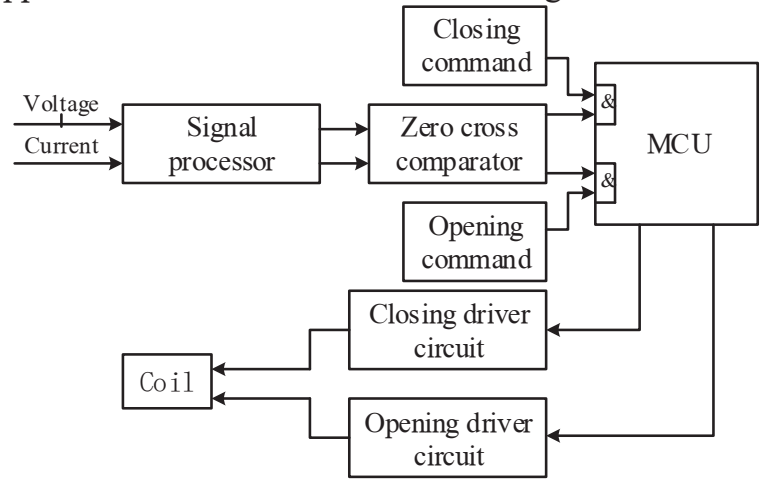

Fig.6 Phase control flow chart

The timing diagram of the phase selection closing strategy is shown in Figure 7. $T_{d c}$ is the time required to calculate and delay in the program. $\mathrm{T}_{\mathrm{cls}}$ is inherent closing time of the contactor. After receiving the closing command, the microprocessor external interrupt is opened, and the zero-crossing comparator will send an interrupt signal to the microprocessor when the voltage is zerocrossed, and the program will calculate $\mathrm{T}_{\mathrm{dc}}$ according to formula (1).

$$
T_{d c}=T-T_{c l s} \bmod \frac{1}{2 f}
$$

Specifically, when the closing action is completed within one cycle, the above formula is simplified to:

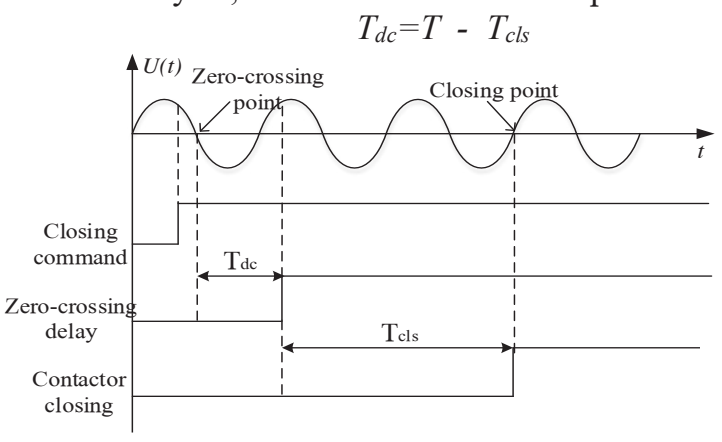

Fig.7 Phase selection timing diagram

\section{Phase selection control system hardware design}

\subsection{Control system hardware circuit overall design}

The microprocessor uses TMS320F2812 as the main control unit to receive the opening and closing command, capture the zero-crossing interrupt and issue the opening and closing drive signal, communicate with the host computer, and control the discharge voltage of the 
capacitor. The peripheral circuit is mainly composed of coil discharge circuit, communication circuit, phase selection control circuit and data acquisition circuit. The overall structure of the designed hardware is shown in Figure 8 .

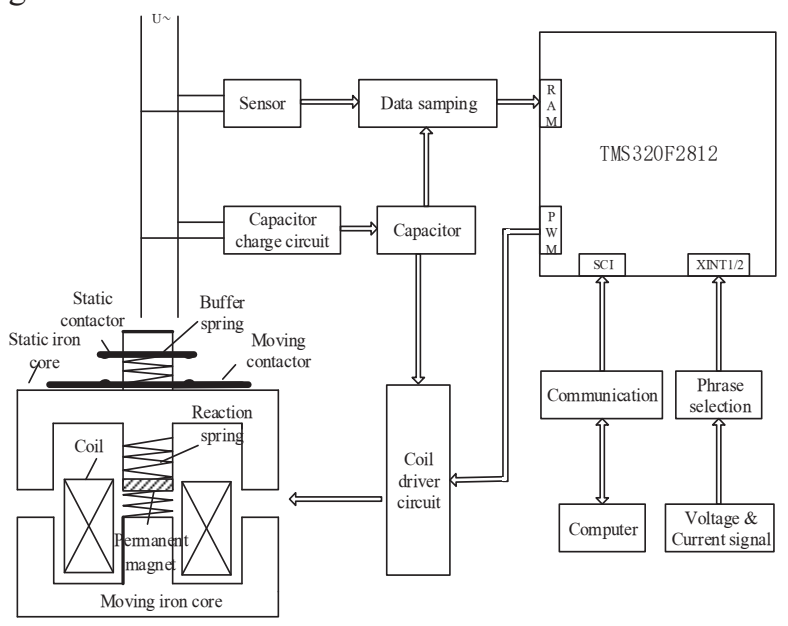

Fig.8 Block diagram of intelligent control system

\subsection{Coil discharge circuit}

The closing and opening operation of the PMA is mainly realized by the discharge of the coil. Figure 9 is a schematic diagram of the designed coil discharge main circuit, where $\mathrm{U}$ is the input AC voltage, which is input through the isolation transformer and is rectified into pulsating DC voltage through the rectifier bridge to charge the storage capacitor $\mathrm{C}_{1}$ through the current limiting resistor $\mathrm{R} ; \mathrm{Q}_{1}$ to $\mathrm{Q}_{5}$ are controllable switching tubes, wherein $\mathrm{Q}_{1}$ is used to control the capacitor charging voltage to a fixed certain value, and Q2 Q5 constitute a bridge circuit, which provides a discharge loop to the coil. Diodes D1 D4 provide a freewheeling circuit for the coil.

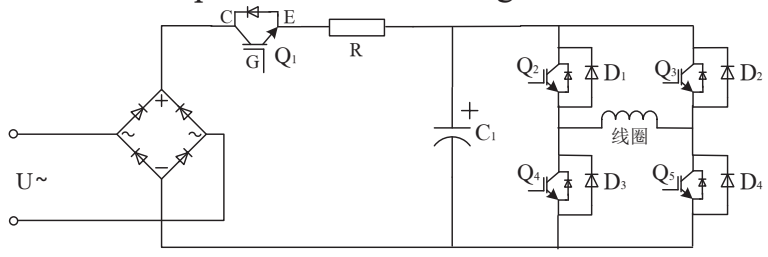

Fig.9 Main circuit for contactor coil discharging

\subsection{Phase selection control module}

The core component of the phase selection system is zerocrossing comparator, and the hardware circuit schematic is shown in Figure 10. The op amp input terminals IN1+ and IN2+ are connected to the voltage signal $\mathrm{U}_{\mathrm{A}}$ and current signal $\mathrm{I}_{\mathrm{A}}$ which are outputted from the transformer, respectively. IN1- and IN2- are connected to the output terminals OUT1 and OUT2 respectively for voltage and current signal following to improve the input impedance and isolation. The output terminals OUT1 and OUT2 are connected to the zero-crossing comparator, and the comparator's terminals OUT1 and OUT2 give the external interrupt signal of the DSP2812 through the two opt couplers U1 and U2, as shown by INT1 and INT2 in the figure. When the input voltage and current signal are greater than 0, INT1 and INT2 will get a low level voltage. Otherwise, a high level voltage will be got so that a square wave signal containing zero crossing points is input to the external interrupt port of the DSP.

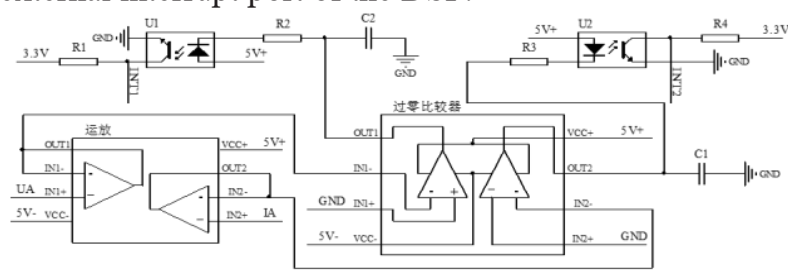

Fig.10 Zero crossing comparator circuit

\subsection{IGBT drive circuit module}

In this paper, the gate driver chip HCPL-316J is selected as the main chip of the IGBT driver circuit. When the IGBT is turned off, its potential is pulled down to $-5 \mathrm{~V}$ to ensure reliable shutdown. It can effectively prevent the IGBT from overvoltage of the input terminal, and turns off the IGBT when the driving voltage is lower than $13 \mathrm{~V}$. The hardware schematic diagram is shown in Figure 11. The chip adopts the input positive terminal VIN+ as the control signal and the negative terminal VIN-ground control mode. Only when VIN+ receives the high level from the DSP, will the internal light of the chip be made. When the voltage value of the VCC2 pin is lower than $13 \mathrm{~V}$, the chip will start the protection function and keep the output terminal VOUT at low level position, and turn off the IGBT in case of the voltage between the gate and the emitter of the IGBT is too low. Exceeding the protection voltage value, the protection circuit in the chip will start to keep the output at low level to turn off the IGBT. At the same time, the FAULT terminal outputs a low level, and sends an error signal to the DSP to protect the working safety of the circuit.

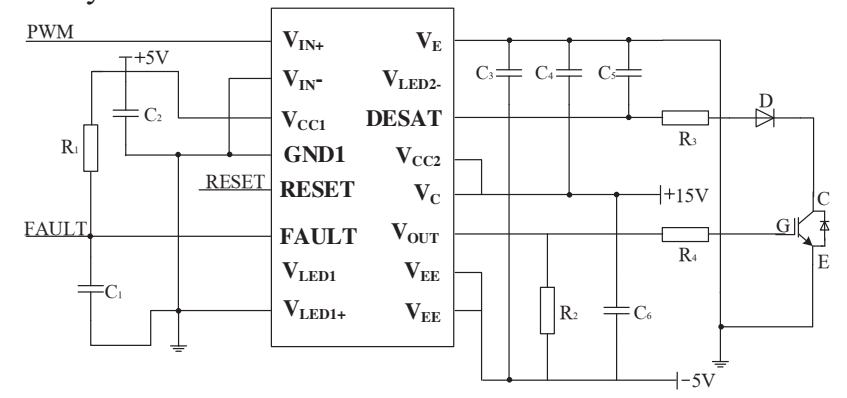

Fig.11 Schematic of HCPL-316J

\section{Phase selection control system software design}

The design of the software system adopts the modular concept and consists of a main program and a subprogram. The subprogram mainly includes a phase selection control subroutine, a data sampling subroutine and a communication subroutine. 


\section{1 main program design}

The main functions of the main program are to complete the initialization of the DSP chip, self-test for each device, allocate the internal memory space of the DSP, and configure and initialize the $\mathrm{I} / \mathrm{O}$ port and each interrupt vector. Figure 12 shows the main program flow chart of the designed software system. After the control system is powered on, the software system starts to initialize, and starts sampling the voltage and current of the three-phase grid. When it detects that the selected phase closing command is sent, it immediately turns on the zero-crossing comparison interrupt, generates an interrupt and calculates the delay time. When the delay time expires, the program enters the opening and closing operation subroutine. Finally, the state and data of the contactor are uploaded to the host computer through the communication subroutine for observation and monitoring, and then return to the data sampling subroutine waiting for the next command signal to arrive.

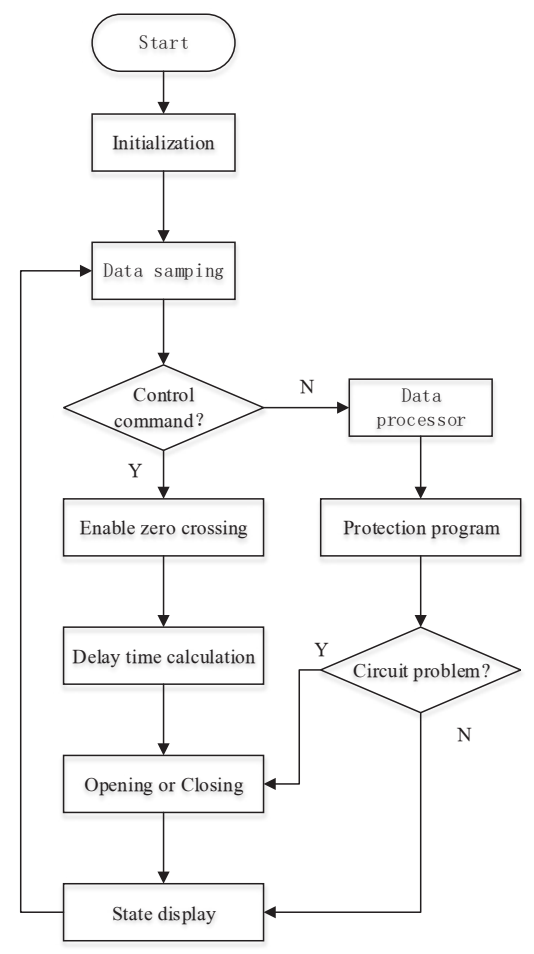

Fig.12 Main program flow chart

\subsection{Phase selection control subprogram}

The phase selection control is the core function of the entire AC contactor intelligent control system. The phase selection control subprogram is shown in Figure 13. After receiving the opening and closing command, the external zero-crossing comparator interrupt is opened. When the voltage and current waveform input signal crosses zero, an interrupt is generated and enters the phase selection control subroutine. The delay time calculation program calculates the delay time required to close at the best point. When the delay is over, the closing and closing command is issued to control the switching tube to be turned on and off. Operate and upload to the host computer to display the current closing status of the contactor. By setting different delay times, the contactor can be able to close at any specified phase angle to switch different loads.

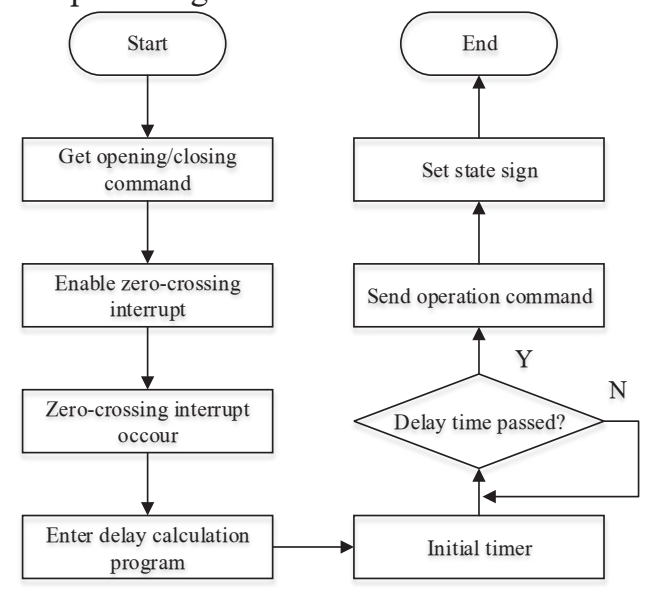

Fig.13 Phase control subroutine flow chart

\section{Test verification}

\subsection{Test platform construction}

On the basis of theoretical analysis and hardware and software design, the test PCB board was fabricated, and the test platform shown in Figure 14 was built, including control board, drive board, host computer, energy storage capacitor, switching power supply and voltage regulator. Based on this test platform, a $12 \mathrm{kV}$ monostable $\mathrm{AC}$ contactor prototype with a permanent magnet operating mechanism was tested, as shown in Figure 15. Through the joint debugging of software and hardware, the phase selection of the contactor are tested, and the intelligent phase control operation of the contactor is realized by writing the corresponding phase selection control strategy in the program.

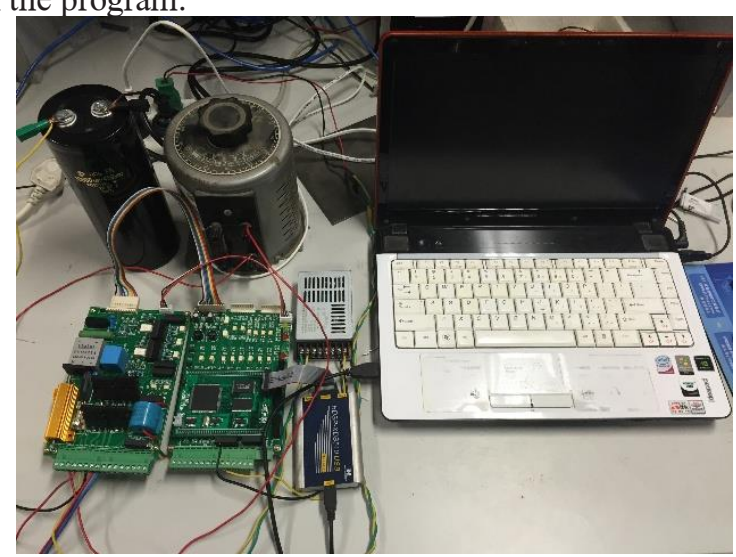

Fig.14 Intelligent control system test platform 


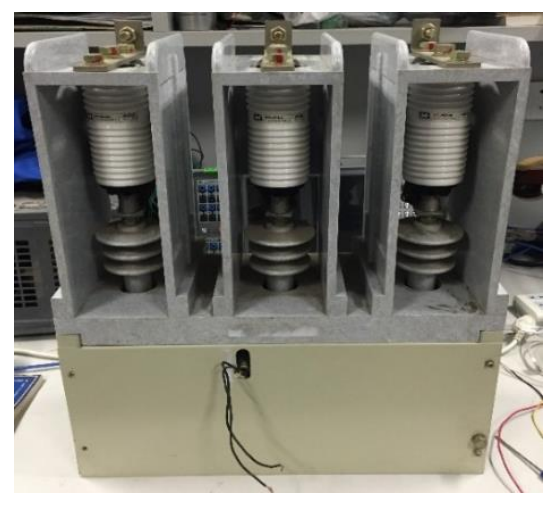

Fig.15 AC contactor prototype

\subsection{Phase selection and closing test}

The principle of moving contact displacement detection is to indirectly indicate the change of displacement by the change of voltage value on the sliding varistor. The circuit consists of a high-precision sliding varistor and a highprecision A/D sampling chip. This project adopts the displacement sensor of the Japanese SAKAE potentiometer 13FLP25A. The sliding jaw of the sensor is fixedly connected with the moving iron core. The displacement variable of the moving iron core is changed into the corresponding resistance value of the sensor, and then the power is supplied by the output power source to convert the resistance value into a voltage value. The change value signal is collected through the ADC sampling channel of the DSP to generate a moving iron core action displacement curve.

The final test results are shown in Figures 16 and 17, which are the closing phase selection control test waveform and the opening phase selection control test waveform respectively. The capacitor voltage is kept at $200 \mathrm{~V}$, and the control contactor operates at the zero crossing point of the voltage signal. The phase control error of the device is within $1^{\circ}$. The current peak value in the coil is about $7 \mathrm{~A}$ when closing. It takes about $27.5 \mathrm{~ms}$ from the start of discharge of the capacitor to the complete closing of the contactor. The actual closing time is about $0.3 \mathrm{~ms}$ away from the zero crossing of the voltage waveform, which is $5.4^{\circ}$ converted to the phase angle. The state change waveform after the contactor is closed is the contact bounce, and the actual closing point is based on the first rising edge waveform; when the contactor is opening, the peak current value in the coil is about 4A, and the capacitor starts to discharge. The time required for the contactor to start opening is about $13 \mathrm{~ms}$, and the actual opening time is about $0.2 \mathrm{~ms}$ from the voltage waveform zero-crossing point, which is $3.6^{\circ}$ converted to a phase angle.

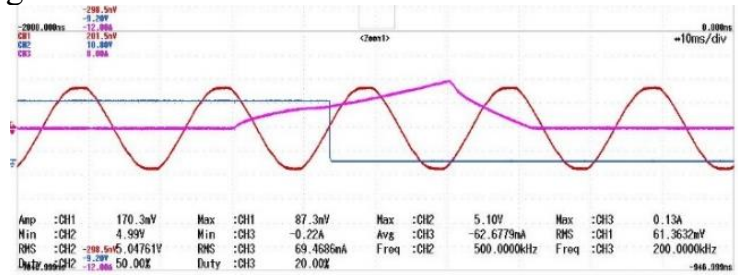

Fig.16 Zero voltage closing test waveform

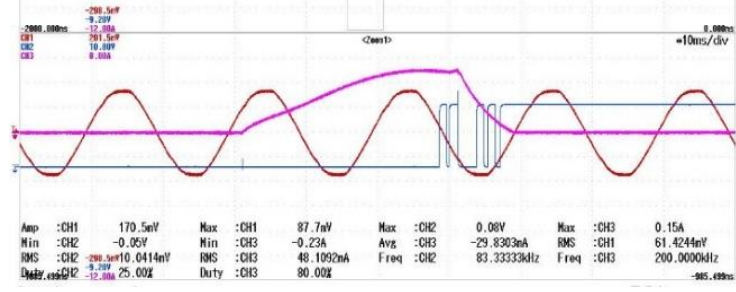

Fig.17 Zero voltage opening test waveform

Maintain the same phase-selection closing strategy and capacitor voltage value, and continuously perform the contactor phase-closing test of the contactor 10 times. The time deviation of the phase angle of the contactor closing and opening and the zero-crossing of the reference voltage current signal is shown in Table 2. The data shows that the phase-selection closing and opening time is within $0.5 \mathrm{~ms}$, and the error is mainly due to the time dispersion of the contactor action. The test results verify the correctness of the theoretical analysis, and the intelligent phase selection control system of the AC contactor is practical.

Table.2. Time deviation of phase selection switching

\begin{tabular}{|c|c|c|c|c|}
\hline time & $\begin{array}{c}\text { Closing } \\
\text { error } \\
\text { time/ms }\end{array}$ & $\begin{array}{c}\text { Corresponding } \\
\text { angle/ }\end{array}$ & $\begin{array}{c}\text { Opening } \\
\text { error } \\
\text { time /ms }\end{array}$ & $\begin{array}{c}\text { Corresponding } \\
\text { angle } \text { / }^{\circ}\end{array}$ \\
\hline 1 & 0.2 & 3.6 & 0.1 & 1.8 \\
\hline 2 & 0.3 & 5.4 & 0.2 & 3.6 \\
\hline 3 & 0.2 & 3.6 & 0.3 & 5.4 \\
\hline 4 & 0.1 & 1.8 & 0.1 & 1.8 \\
\hline 5 & 0.1 & 1.8 & 0.2 & 3.6 \\
\hline 6 & 0.3 & 5.4 & 0.2 & 3.6 \\
\hline 7 & 0.4 & 7.2 & 0.2 & 3.6 \\
\hline 8 & 0.3 & 5.4 & 0.2 & 3.6 \\
\hline 9 & 0.2 & 3.6 & 0.1 & 1.8 \\
\hline 10 & 0.3 & 5.4 & 0.2 & 3.6 \\
\hline
\end{tabular}

\section{Conclusion}

The PMA of the AC contactor and its intelligent phase selection control system are studied is this paper. The PMA is designed, and the dynamic characteristics of the actuator are simulated and analyzed in ANSOFT to meet the performance requirements of phase selection control. Based on the analysis of phase selection control principle and phase selection strategy of power capacitors, the software and hardware of intelligent phase selection control system are designed respectively, including coil discharge circuit, IGBT diver circuit, phase selection control circuit and communication circuit. The test is verified on the $12 \mathrm{kV}$ permanent magnet monostable $\mathrm{AC}$ contactor of the operating mechanism. The results show that the designed $\mathrm{AC}$ contactor intelligent phase selection control system can control the phase-selection closing precision within $1 \mathrm{~ms}$, which can effectively reduce the contactor inrush current and overvoltage generated during the closing and opening operation, improve the electrical life of the contactor and have significant engineering practical significance. 


\section{References}

1. H. Lin, X. Wang, S. Fang, P. Jin, S. Ho, Design optimization and intelligent control of permanentmagnet contactor (IEEE Trans. Ind. Electron., vol. 60, no. 11, pp. 5148-5159, 2013)

2. X. Wang, S. Ni, H. Lin, S. Fang, P. Jin, Control technique of selection phase angle for intelligent permanent magnet contactor (Electric Machines and Control, vol. 16, no. 10, pp. 63-70,2012)

3. Z. Xu, Z. Cao, P. Zhang, New Type of Intelligent AC Contactors for Making and Breaking Condenser Bank (Low Voltage Apparatus, vol. 1, no. 39, pp. 2023,2006)

4. Z. Xu, P. Zhang, Dynamic Optimal Design Of Intelligent Ac Contactor In The Whole Course (Proceedings Of The Chinese Society For Electrical Engineering, vol. 25, no. 17, pp. 156-161,2005)

5. F. Guo, Choose Phase to Close Technology Research of AC Contactor (Electrotechnical Journal, vol. 2, no. 10, pp. 33-37, 2004)

6. X. Wang, H. Lin, S. Fang, P. Jin, Analysis of Displacement Subsection PWM Control and Dynamic Characteristic for Closing Process of Permanent Magnet Contactor (Electric Machines and Control, vol. 30, no. 3, pp. 113-118, 2010)

7. P. Dai, Y. Zhou, Z. Sun, Y. Geng, Research on Intelligent Control of Segmented Voltage for AC Contactor Based on Current Variation (Low Voltage Apparatus, vol. 6, no. 23, pp. 7-11, 2011)

8. H. Huang, G. Wu, Z. Wu, C. Chen, Realization and Application on AC Contactor Life Testing System Based on Phase-controlled Technology (Journal of Wenzhou University (Natural Sciences), vol. 37, no. 2, pp. 27-32,2016) 